\title{
Ber Variation in an uwb ofdm mimo Communications System Based on Measurements made in a Picocell Wireless Environment
}

\author{
Pat Chambers \\ Technological University Dublin \\ Conor Downing \\ Technological University Dublin, conor.downing@tudublin.ie \\ Hussein Baher \\ Technological University Dublin
}

Follow this and additional works at: https://arrow.tudublin.ie/engschececon

Part of the Electrical and Computer Engineering Commons

\section{Recommended Citation}

Chambers, P., Downing, C., Baher, H.: Ber variation in an uwb ofdm mimo communications system based on measurements made in a picocell wireless environment. First European Conference on Antennas and Propagation, 2006. pp1-6. EuCAP 2006. doi:10.21427/y1cv-1221

This Conference Paper is brought to you for free and open access by the School of Electrical and Electronic Engineering at ARROW@TU Dublin. It has been accepted for inclusion in Conference papers by an authorized administrator of ARROW@TU Dublin. For more information, please contact arrow.admin@tudublin.ie, aisling.coyne@tudublin.ie,gerard.connolly@tudublin.ie.

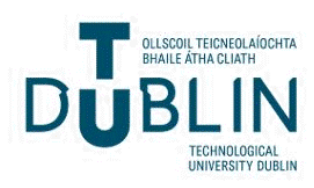




\title{
BER VARIATION IN AN UWB OFDM MIMO COMMUNICATIONS SYSTEM BASED ON MEASUREMENTS MADE IN A PICOCELL WIRELESS ENVIRONMENT.
}

\author{
Pat Chambers, Conor Downing, and Hussein Baher \\ Dublin Institute of Technology, Kevin Street, Dublin 8, Ireland.
}

\begin{abstract}
A picocell UWB MIMO communications system model is considered which has five orthogonal sub-channels, each with its own OFDM signal. The effect of removing the various channel impulse response components which arise due to the multi-path propogation, a phenomenon characteristic of wide band transmission, is considered. This is a measurement based analysis where the measurements, themselves, were made, in a specially constructed enclosure which had reflective surfaces, using specially designed UWB antennas.
\end{abstract}

Key words: UWB; MIMO; OFDM; BER.

\section{INTRODUCTION}

In the context of antenna communications systems, the multiple-input/multiple-output (MIMO) communications system model as well as the ultra wide band (UWB) communications channel have received a lot of attention lately. In conjunction with orthogonal frequency division multiplexing (OFDM), the MIMO communications system model can exploit the multi-path signal propagation which is characteristic of the UWB communications systems. Primarily, the analysis made herein seeks to demonstrate the effect of the various components of the multi-path signal as detected at the receiver on the MIMO communications system performance. More specifically, the removal or perhaps obstruction of each of the multipath components is examined here in terms of the bit error rate (BER) of the MIMO communications system model.

Previous work by the authors [1] described a single carrier analysis, based also on UWB measurements, where the convenient formula for generalised capacity as outlined by Foschini and Gans [2] was used to calculate the potential capacity of a $5.2 \mathrm{GHz}$ carrier frequency within the UWB band. This approach indicated an increase in the potential capacity of this single carrier frequency when the line of sight (LOS) signal path was removed from the calculation for the channel transfer function matrix. It could be argued, however, that in the context of that approach, that there may have been a temporal diversity gain due purely to the normalisation procedure of the channel transfer function matrix.

For this analysis, measurements of the UWB channel were made using an Anritsu 37369A vector microwave network analyser (VMNA). Although various definitions of the UWB exist [3], the UWB channel measurements here were made within a band between $3.1 \mathrm{GHz}$ and $10.6 \mathrm{GHz}$. These measurements were then incorporated into a 256 sub-band MIMO OFDM simulation where an investigation of the multi-path environment then proceeds without the need for the normalisation discussed. It should be noted that in order to simplify analysis, a sub-band bandwidth of approximately $15 \mathrm{MHz}$ was assumed so that one complete OFDM communications systems could be implemented with respect to the UWB defined. This is in contrast to work by Siriwonpairot et al. [4] where the $7.5 \mathrm{GHz}$ UWB band is split into separate $500 \mathrm{MHz}$ channels, each with their own OFDM signal. In effect, it is assumed in this context that any spectral null observed in this work will have a bandwidth greater then $15 \mathrm{MHz}$.

\section{OFDM COMMUNICATIONS SYSTEMS}

One of the main problems associated with transmission over the UWB channel is 'multi-path fading'. As a result of the multi-path environment, there can exist significant losses in received power at certain frequencies for a given UWB channel. A possible solution to this problem is to transmit data in many different sub-bands. This has a clear advantage over singlecarrier techniques, but leads to another problem of interband interference (IBI). Orthogonal frequency division multiplexing (OFDM) avoids IBI by utilising the fast fourier transform (FFT) to create a series of orthogonal sub-bands within the communications channel. OFDM can then be further improved by coding techniques, precoding techniques and spreading techniques. However, to simplify analysis here, the OFDM technique used is conventional and is described below. It should be noted that the following analysis will be extended to an UWB MIMO OFDM communications system in the next section. 
At the transmitter, a frame of data or 'symbol' is transmitted which is seen to consist of a number sub-symbols which, in turn, are the outputs from a bank of quadrature amplitude modulators. Each of the quadrature amplitude modulation (QAM) constellations over the band of frequencies on the communications channel is thought of as being complex and of equal constellation size or 'M-ary number'. They each form a variable, $\mathbf{x}_{\mathbf{n}}$. A complete frequency domain vector of channel inputs, $\mathbf{X}$ known as the 'symbol' described, is defined by equation (1) below and is derived from each QAM constellation variable, $\mathbf{x}_{\mathbf{n}}$ :

$$
\mathbf{X}=\left[\mathbf{x}_{\mathbf{0}}, \mathbf{x}_{\mathbf{1}}, \ldots \mathbf{x}_{\mathbf{n}-\mathbf{1}}, 0, \mathbf{x}_{\mathbf{n}-\mathbf{1}}^{\dagger}, \mathbf{x}_{\mathbf{n}-\mathbf{2}}^{\dagger}, \mathbf{x}_{\mathbf{1}}^{\dagger}\right](1)
$$

In the context of the sub-script notation above, transmission occurs over $2 \mathbf{n}$ sub-bands, where generally, $2 \mathbf{n}=$ $512=N$. Each transmitted symbol thus contains 512 sub-symbols and $\mathbf{X}$ is an $N \times 1$ vector. The notation, ' $\dagger$ ' refers to the complex conjugate of a given variable and so the vector, $\mathbf{X}$ is composed of a series of elements which are conjugate symmetric about the origin. The element, $\mathbf{x}_{\mathbf{0}}$ is always assigned a value of 1 . The output of the OFDM transmitter is a time domain signal, which results from taking the inverse fast Fourier transform (IFFT) of the frequency domain vector of channel inputs, $\mathbf{X}$. This time domain signal will, in turn, be entirely real owing to the conjugate symmetry of $\mathbf{X}$.

The fast Fourier transform (FFT) may be written as a unitary matrix of elements, $\mathbf{W}$, and is defined below in equation (2) as a $k \times l$ matrix:

$$
\mathbf{W}_{\mathbf{k}, \mathbf{l}}=\frac{1}{N} \exp \left\{-j \frac{2 \pi}{N} k l\right\}
$$

In this case $j=\sqrt{-1}$ and if a vector of dimensions $N \times 1$ is to be transformed, then $0 \leq k, l \leq N-1$. The quantity $\frac{1}{N}$ may be thought of as a scaling factor which is relevant to this context. The corresponding IFFT matrix operation is the Hermitian transpose of $\mathbf{W}$, written as $\mathbf{W}^{\mathbf{H}}$. OFDM can exploit the fact that a circulant matrix, $\widetilde{\mathbf{H}}_{\text {UWB }}$ is diagonalised by the $\mathbf{W}$ and $\mathbf{W}^{\mathbf{H}}$ matrix operations. When these operations have been performed with respect to $\widetilde{\mathbf{H}}_{\mathbf{U W B}}$, there is a significant reduction in the interband interference (IBI) that would normally occur as result of the communications channel. An expression for the diagonal matrix, $\boldsymbol{\Lambda}$, that arises from these operations is given by equation (3) below:

$$
\Lambda=\mathbf{W} \widetilde{\mathbf{H}}_{\mathbf{U W B}} \mathbf{W}^{\mathbf{H}}
$$

In practice, the frequency domain vector of channel inputs, $\mathbf{X}$, has a series of redundant sub-symbols known as a 'cyclical prefix',(CP) affixed to it. When the time domain samples derived from $\mathbf{X}$, by means of the matrix operation $\mathbf{W}^{\mathbf{H}}$ at the transmitter output, are then convolved with the channel impulse response, the channel impulse itself appears as a circulant matrix multiplication, $\widetilde{\mathbf{H}}_{\mathbf{U W B}}$, on $\mathbf{X}$ at the receiver. The length of the

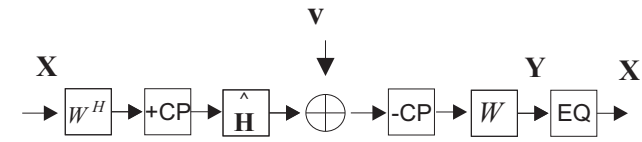

Figure 1. A single channel OFDM communications system.

$\mathrm{CP}$ is determined with respect to the delay spread of the channel. In this regard, the CP also reduces intersymbolinterference (ISI) by mitigating the effect of the length in time of the channel impulse response on the symbol, $\mathbf{X}$. As indicated, the information content of the $\mathrm{CP}$ is ignored at the receiver. In this way, $\widetilde{\mathbf{H}}_{\mathrm{UWB}}$ is the circular convolution matrix, which is derived from a vector containing a discrete time measurement of the UWB channel, when OFDM is applied to the channel. An $N \times 1$ vector of frequency domain channel outputs, $\mathbf{Y}$, may be derived at the receiver by means of a further matrix multiplication by $\mathbf{W}$. An expression for $\mathbf{Y}$ is given by equation (4) below:

$$
\mathbf{Y}=\mathbf{W}\left\{\widetilde{\mathbf{H}}_{\mathbf{U W B}} \mathbf{W}^{\mathbf{H}} \mathbf{X}+\mathbf{v}\right\}
$$

$\mathbf{v}$ is an $N \times 1$ added white Guassian noise (AWGN) vector. Substituting equation (3) into equation (4) allows $\mathbf{Y}$ to then be written as equation (5) below:

$$
\mathbf{Y}=\mathbf{\Lambda} \mathbf{X}+\mathbf{v}^{\prime}
$$

where

$$
\mathbf{v}^{\prime}=\mathbf{W} \mathbf{v}
$$

$\mathbf{v}^{\prime}$ refers to the $N \times 1$ vector of AWGN $\mathbf{v}$, which has been transformed into the frequency domain by the matrix multiplication in equation (6) above. The frequency domain vector $\mathbf{Y}$ has a gain profile, $\boldsymbol{\Lambda}$ with respect to the original frequency domain channel input vector $\mathbf{X}$, as well as having an additional noise vector $\mathbf{v}^{\prime} . \boldsymbol{\Lambda}$ is a diagonal matrix and in this way ensures that there is little or no IBI seen at receiver. Inverting each individual element along the main diagonal to form an $N \times 1$ vector, $\mathbf{D}$, would allow the recovery of the original vector of frequency domain inputs to the channel, i.e., X. An expression for the vector $\mathbf{D}$ is given by equation (7) below:

$$
\mathrm{D}=\left[\frac{1}{\Lambda_{1,1}}, \frac{1}{\Lambda_{2,2}}, \frac{1}{\Lambda_{3,3}}, \ldots, \frac{1}{\Lambda_{\mathrm{N}, \mathrm{N}}}\right]
$$

OFDM with respect to a single communications channel is presented in figure (4) below. Equalisation, i.e., 'EQ', refers to the recovery of $\mathbf{X}$ at the receiver.

\section{THE UWB MIMO OFDM COMMUNICA- TIONS SYSTEM MODEL}

Referring to figure (2), it is clear from this measurement of the channel impulse that a calculation of the delay spread is possible. Specifically figure (2) shows a line of sight (LOS) component, two signals which have undergone one reflection before being detected at the receiver, 


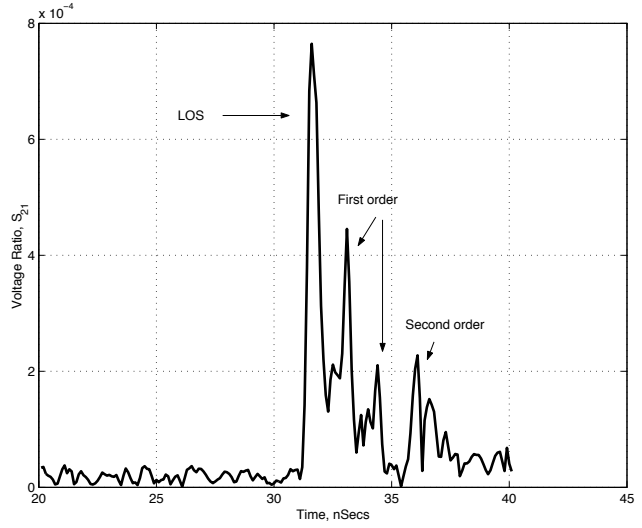

Figure 2. A time-domain measurement, with respect to the UWB bandwidth defined, of the channel impulse response which results from a multi-path environment.

i.e. two first order components and finally a signal which has undergone two reflections before detection or second order component. Of course, it is knowledge of the physical dimensions of the measurement environment which allows for such deductions to be made. Details of measurements will be discussed in the next section.

Given measurements similar to figure (2) which are indicative of a $5 \times 5 \mathrm{MEA}$ of transmit and receive antennas, a suitable MIMO communications system model may now be defined. For each possible permutation of transmitter and receiver positioning outlined above, there is a measurement of $S_{21}$ similar to figure (2) which is a 201 point IFFT of the UWB frequency response of the channel which exists between each permutation described. Making the assumption that the UWB frequency response represents a complete impulse response of a given single channel, then convolution of a given time domain signal with this 201 point IFFT allows for the comparison of two frequency domain gains which in the context of the model presented in figure (1) would be $\mathbf{X}$ and $\mathbf{Y}$. The 201 point IFFT can be expanded to a 512 point IFFT by means a spline algorithm allowing the model for OFDM outlined in the previous section to be relevant to this context here. Taking the element-by-element division of $\mathbf{X}$ and $\mathbf{Y}$ allows for the frequency response of the channel to be derived. Figure (3) below indicates how this same process might be used to calculate the frequency response of the four such permutations that would exist in a $2 \times 2$ MEA of transmit and receive antennas. The $2 \times 2$ case is now examined for the sake of clarity. As indicated previously this work focuses on a $5 \times 5$ case.

The simulation first generates a series of QAM signals at the two inputs, i.e. the vectors $\mathbf{X}_{1}$ and $\mathbf{X}_{2}$. With respect to these QAM signals, it then performs the various matrix multiplications relevant to the terms: $\mathbf{W}^{\mathbf{H}}$, $\mathbf{W}, \widetilde{\mathbf{H}}_{\mathrm{UWB}(1,1)}, \widetilde{\mathbf{H}}_{\mathrm{UWB}(\mathbf{1}, 2)}, \widetilde{\mathbf{H}}_{\mathrm{UWB}(2,1)}$ and $\widetilde{\mathbf{H}}_{(\mathbf{2}, 2)}$. Each of the matrices: $\widetilde{\mathbf{H}}_{\mathbf{U W B}(\mathbf{1}, \mathbf{1})}, \widetilde{\mathbf{H}}_{\mathbf{U W B}(\mathbf{1}, \mathbf{2})}$, $\widetilde{\mathbf{H}}_{\mathrm{UWB(2,1)}}$ and $\widetilde{\mathbf{H}}_{\mathrm{UWB}(\mathbf{2 , 2})}$ is a circulant matrix which is related to a vector containing a set of points not unlike

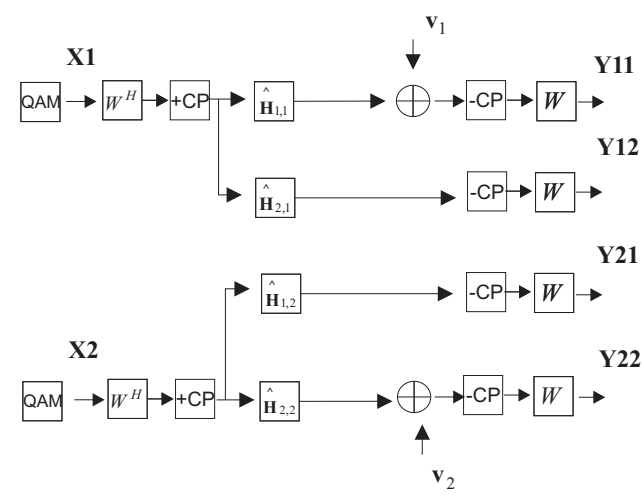

Figure 3. Model for the measurement based simulation used to calculate the frequency response of all the channels or permutations in a $2 \times 2 M E A$ of transmit and receive antennas.

what was plotted in figure (2). Four $N \times 1$ frequency domain output vectors are now introduced: $Y 11_{N}, Y 12_{N}$, $\mathbf{Y} 21_{\mathbf{N}}$ and $\mathbf{Y} 22_{\mathbf{N}}$. A three dimensional array, $\mathbf{H}_{\omega(\mathbf{i}, \mathbf{j}, \mathbf{N})}$ may be calculated. For this particular $2 \times 2$ MEA of transmit and receive antennas: $i=1,2$ and $j=1,2$. At this point, the notation, $(\cdot)_{\omega}$, should be noted. It indicates that each $2 \times 2$ matrix contained within the three-dimensional array, $\mathbf{H}_{\omega(\mathbf{i}, \mathbf{j}, \mathbf{N})}$, contains elements which are frequency dependent. For example, $\mathbf{H}_{\omega(\mathbf{1}, \mathbf{1}, \mathbf{N})}$ is an $N \times 1$ vector containing the element-by-element division of the $N \times 1$ vectors of $\mathbf{X}_{\mathbf{1}}$ into $\mathbf{Y}_{\mathbf{1 1}}$ and as indicated previously, $\mathbf{X}_{\mathbf{1}}$ and $\mathbf{Y}_{11}$ are both frequency domain vectors. Similar expressions for $\mathbf{H}_{\omega(\mathbf{1}, \mathbf{2}, \mathbf{N})}, \mathbf{H}_{\omega(\mathbf{2}, \mathbf{1}, \mathbf{N})}$ and $\mathbf{H}_{\omega(\mathbf{2}, \mathbf{2}, \mathbf{N})}$ may also be derived and expressions for all of these are given below in equations (8), (9), (10) and (11) respectively:

$$
\begin{gathered}
\mathbf{H}_{\omega(1,1, N)}=\left[\frac{\mathrm{Y} 11_{1}}{\mathrm{X} 1_{1}}, \frac{\mathrm{Y} 11_{2}}{\mathrm{X} 1_{2}}, \frac{\mathrm{Y} 11_{3}}{\mathrm{X} 1_{3}}, \ldots, \frac{\mathrm{Y} 11_{\mathbf{N}}}{\mathrm{X} 1_{\mathrm{N}}}\right] \\
\mathbf{H}_{\omega(1,2, \mathrm{~N})}=\left[\frac{\mathrm{Y} 12_{1}}{\mathrm{X} 1_{1}}, \frac{\mathrm{Y} 12_{2}}{\mathrm{X} 1_{2}}, \frac{\mathrm{Y} 12_{3}}{\mathrm{X} 1_{3}}, \ldots, \frac{\mathrm{Y} 12_{\mathrm{N}}}{\mathrm{X} 1_{\mathrm{N}}}\right] \\
\mathbf{H}_{\omega(2,1, \mathrm{~N})}=\left[\frac{\mathrm{Y} 21_{1}}{\mathrm{X} 2_{1}}, \frac{\mathrm{Y} 21_{2}}{\mathrm{X} 2_{2}}, \frac{\mathrm{Y} 21_{3}}{\mathrm{X} 2_{3}}, \ldots, \frac{\mathrm{Y} 21_{\mathrm{N}}}{\mathrm{X} 2_{\mathrm{N}}}\right] \\
\mathbf{H}_{\omega(2,2, \mathrm{~N})}=\left[\frac{\mathrm{Y} 22_{1}}{\mathrm{X} 2_{1}}, \frac{\mathrm{Y} 22_{2}}{\mathrm{X} 2_{2}}, \frac{\mathrm{Y} 22_{3}}{\mathrm{X} 2_{3}}, \ldots, \frac{\mathrm{Y} 22_{\mathrm{N}}}{\mathrm{X} 2_{\mathrm{N}}}\right]
\end{gathered}
$$

The calculation of the three dimensional array, $\mathbf{H}_{\omega(\mathbf{i}, \mathbf{j}, \mathbf{N})}$, within the simulation may be thought of as being equivalent to a channel sounding sequence. At this point, $N$ singular value decompositions (SVD)s are performed on each of the $N 2 \times 2$ matrices $\mathbf{H}_{\omega(\mathbf{i}, \mathbf{j})}$ which may be de-

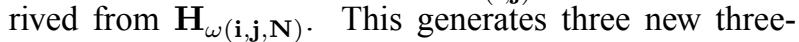
dimensional arrays: $\mathbf{U}_{\mathbf{i}, \mathbf{j}, \mathbf{N}}, \mathbf{S}_{\mathbf{i}, \mathbf{j}, \mathbf{N}}$ and $\mathbf{V}_{\mathbf{i}, \mathbf{j}, \mathbf{N}}$. The relationship between the $2 \times 2$ matrices within these arrays may be described by an equation similar to equation (3), i.e. equation (32) below:

$$
\mathbf{S}_{\mathbf{i}, \mathbf{j}, \mathbf{N}}=\mathbf{U}_{\mathbf{i}, \mathbf{j}, \mathbf{N}}^{\mathbf{H}} \mathbf{H}_{\omega(\mathbf{i}, \mathbf{j}, \mathbf{N})} \mathbf{V}_{\mathbf{i}, \mathbf{j}, \mathbf{N}}
$$




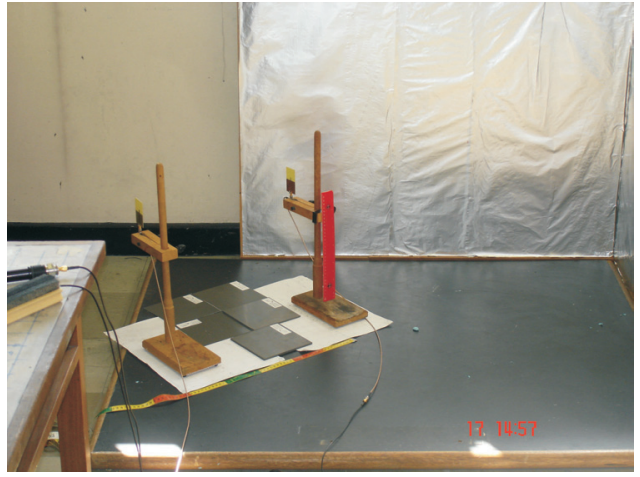

Figure 5. The experimental set-up.

Clearly, there are now $N$ sets of matrices: $\mathbf{U}_{\mathbf{i}, \mathbf{j}}, \mathbf{S}_{\mathbf{i}, \mathbf{j}}$ and $\mathbf{V}_{\mathbf{i}, \mathbf{j}}$. Each matrix $\mathbf{U}_{\mathbf{i}, \mathbf{j}}$ and $\mathbf{V}_{\mathbf{i}, \mathbf{j}}$ is a unitary matrix with $\mathbf{U}_{\mathbf{i}, \mathbf{j}}^{\mathbf{H}}$ being the hermitian transpose of $\mathbf{U}_{\mathbf{i}, \mathbf{j}} . \mathbf{S}_{\mathbf{i}, \mathbf{j}}$ is a diagonal matrix containing the singular values of $\mathbf{H}_{\omega(\mathbf{i}, \mathbf{j})}$.

Figure (4) shows the MIMO Communications channel. The overall gain profile of the entire $2 \times 2$ communications system would assume that given by matrix $\mathbf{S}_{\mathbf{i}, \mathbf{j}}$. Specifically in the context of the simulation, the two $N \times 1$ vectors: $\mathbf{X} \mathbf{1}$ and $\mathbf{X} \mathbf{2}$ are multiplied by four $N \times 1$ vectors, $\mathbf{U}_{1,1}, \mathbf{U}_{1,2}, \mathbf{U}_{\mathbf{2}, 1}$ and $\mathbf{U}_{\mathbf{2}, 2}$ which are derived from appropriate partitioning of the three dimensional matrix: $\mathbf{U}_{\mathbf{i}, \mathbf{j} \mathbf{H}}$. The two $N \times 1$ vectors: $\mathbf{Y} \mathbf{1}$ and $\mathbf{Y} \mathbf{2}$ are multiplied by four similar $N \times 1$ vectors derived from the three dimensional matrix $\mathbf{V}_{\mathbf{i}, \mathbf{j}}$. Figure 4 gives a complete description of this process, in the context of the simulation model. It should be noted that SIMULINK [7] was used to run the simulation and that the added noise vectors, $\mathbf{v}_{\mathbf{1}}$ and $\mathbf{v}_{\mathbf{2}}$, were such that an SNR of $18 \mathrm{~dB}$ arose on each sub-channel.

\section{UWB MEASUREMENTS}

Experimental results were obtained in a specially constructed enclosure which consisted of two highly reflective surfaces at right angles to each other. Other surfaces were covered by microwave absorbing material. This reduced the number of paths to one direct path, two singly reflected or first order paths, and one doubly reflected or second order path. Four multi-path signals were identified on an MNA in the time domain. The experimental set-up is shown in figure 5. Two antennas were placed $36 \mathrm{~cm}$ apart, this distance was deemed adequate for making measurements in the far-field regime with respect to a frequency of $5.2 \mathrm{GHz}(\lambda=5.77 \mathrm{~cm})$. As stated, the 5.2 $\mathrm{GHz}$ carrier frequency was the focus of previous work by authors in the context of a single carrier calculation. It is chosen as the separation here for no reason in particular, other then the fact that it is a sensible antenna spacing. Detection of the multi-path environment in the time domain was thus made with respect to an IFFT of the frequency range extending from $3.1 \mathrm{GHz}$ to $10.6 \mathrm{GHz}$.

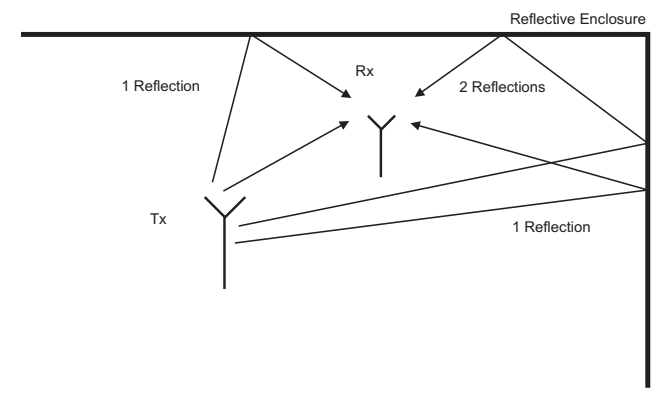

Figure 6. A sketch of the specially built enclosure identifying multi-path components with respect to one transmit element, Tx, and one receive element, $R x$. 1st order signal paths undergo one reflection and second order signal paths undergo two reflections within the enclosure..

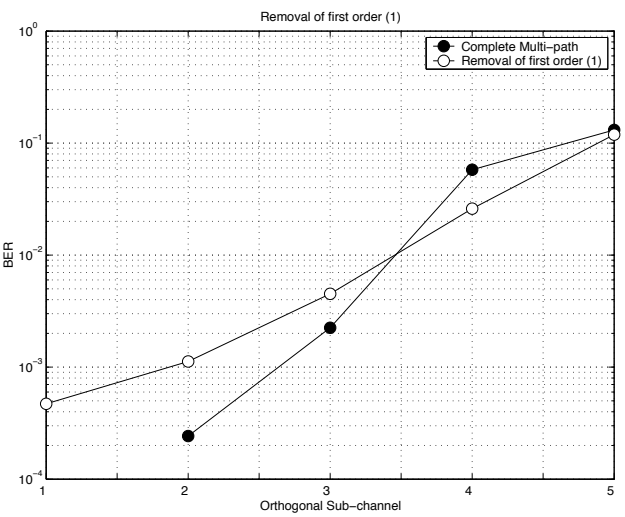

Figure 7. Removal of first order (1)

A geometrical appreciation of the experimental set-up, shown in figure 6 , confirms that LOS, first order and second order components can be observed in figure 2

\section{RESULTS}

Figures 7, 8, 9 and 10 reflect the analysis of the UWB MIMO OFDM communications system simulation. The emphasis here is a little different to the single carrier results presented [1]. The following results refer to a $5 \times 5$ MEA of transmitters and receivers within an UWB MIMO OFDM communications system. In each figure, the $\mathrm{x}$-axis refers to the BER recorded when this $5 \times 5$ MIMO communications system is viewed as a series of separate orthogonal sub-channels. The simulation randomly generates at total of 329,450 bits and as a result of the five orthogonal sub-channels within the simulation model of the $5 \times 5$ MIMO communications system, as described previously, this amount of bits is divided equally amongst the five sub-channels. The BER of each subchannel is then found by comparing each of the 65,890 bits at the transmit and receive end of each sub-channel. 


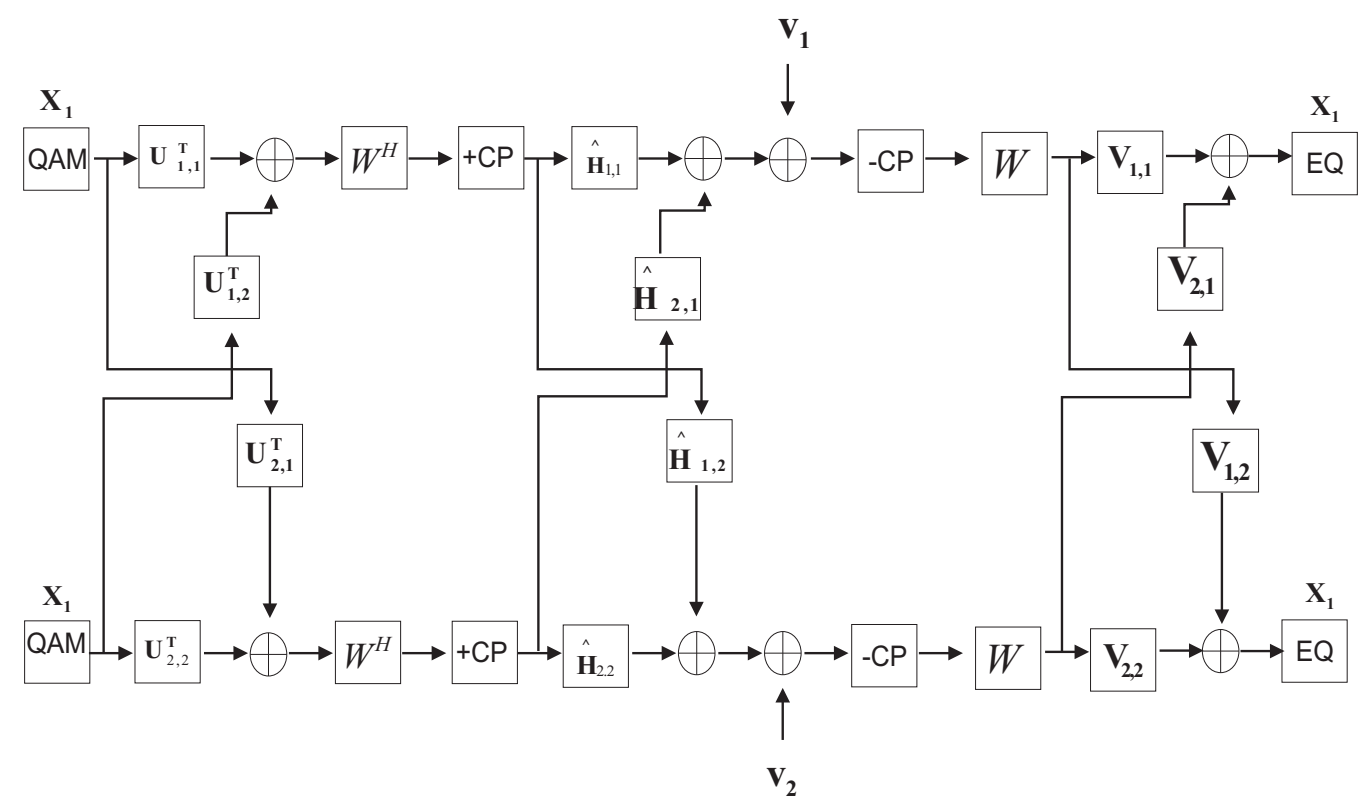

Figure 4. Model for the measurement based simulation of a MIMO communications system comprising of a $2 \times 2$ MEA of transmit and receive antennas.

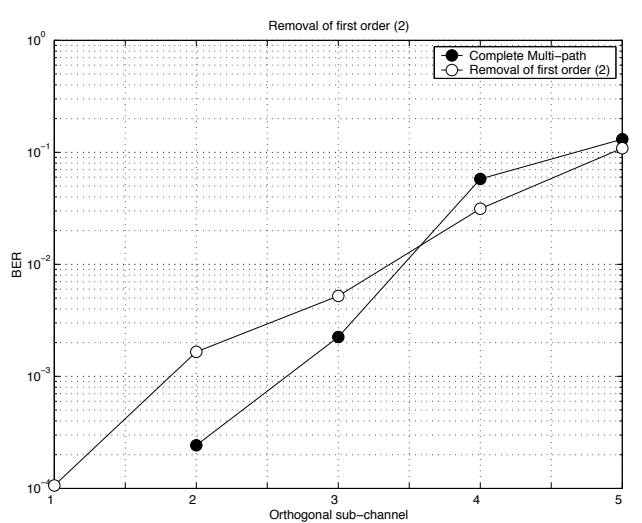

Figure 8. Removal of first order (2)

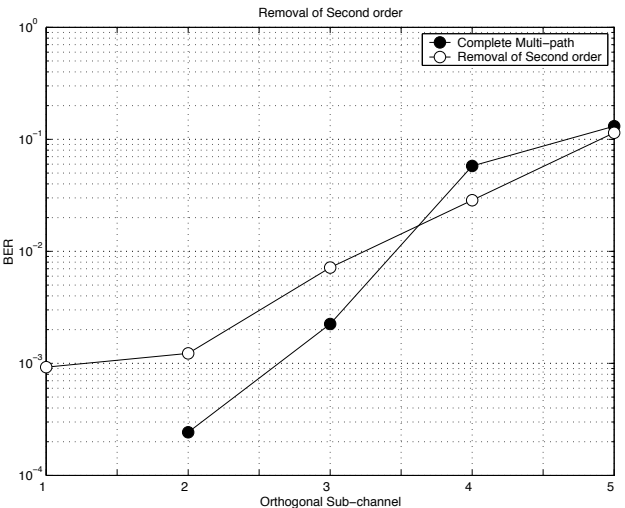

Figure 9. Removal of second order 


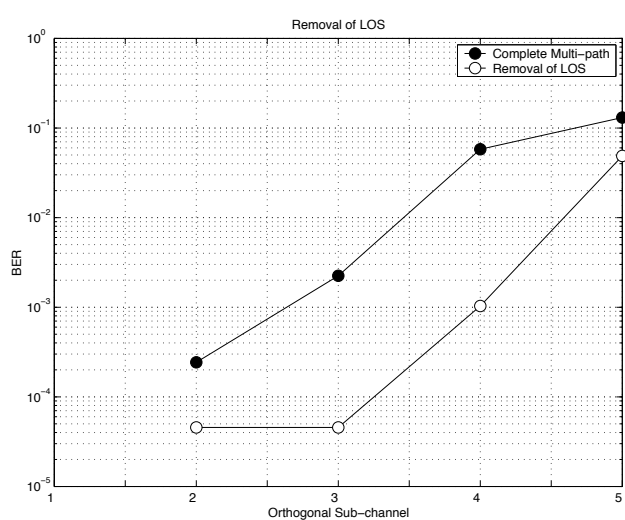

Figure 10. Removal of LOS formance. This idea has also been suggested by Gespert in a more analytical fashion to here [8].

\section{ACKNOWLEDGEMENTS}

The authors would like to thank Dr. Max Ammann and Matthias John of the Centre for Telecommunications Value-Chain Research (CTVR) for providing specially designed UWB Antennas for this work [9].

The authors would also like to thank Dr. Gerry Farrell and Tony Grennan of DIT as well as Barry Cardiff of S3 Group for their helpful comments.

\section{REFERENCES}

[1] P., Chambers, C., Downing, H., Baher. "Capacity Variation in a MIMO Picocell Wireless Link Based on UWB Measurments.". Irish Signals and Systems Conference (ISSC), Dublin Institute of Technology (DIT), June 2006.

[2] G.F., Foschini, M.J., Gans. "On Limits of Wireless Communication in a Fading Environment When Using Multiple Antennas". Wireless Personal Communications, Volume 6, no.3 pp 311-335, March 1995.

[3] F., Sabath, E.L., Mokole. "Definitions and Classification of Ultra-Wideband Signals and Devices". The Radio Science Bulletin, No. 310, pp 12-26, June 2005.

[4] W.P., Siriwonpairat, W., Su, M., Olfat, K.J., Ray Liu. "Multiband-OFDM MIMO Coding Framework for UWB Communications Systems". IEEE Transactions on Signal Processing, Vol. 54, No.1 pp 214-224, January 2006.

[5] P. Chambers, C. Downing, H. Baher. "Bandwidth, Spectral Efficiency and Capacity Variation in TwistedPair Cable.”. Irish Signals and Systems conference, Queen's University, Belfast 2004.

[6] G.D., Durgin. "Space-Time Wireless Channels.". Prentice Hall Communications Engineering and Emerging Technologies Series, Chapter 8, pp 205-232, Pearson Education 2003.

[7] MATLAB/SIMULINK “www.themathworks.com.”. Accessed, 25 March, 2006.

[8] D., Gesbert. "From Theory to Practice: An Overview of MIMO Space-Time Coded Wireless Systems". IEEE Journal on Selected Areas in Communications, Vol. 21, pp. 281-291, April, 2003.

[9] M.J., Ammann, M., John. "Optimum design of the printed strip monopole". Antennas and Propagation Magazine, IEEE, Volume 47, Issue 6, Dec. 2005 pp 59-61. 